\title{
Imaging in Latest Dentistry - A Review
}

\author{
Dr.Yesh Sharma ${ }^{1}$, Dr. Jasmine Marwaha ${ }^{2}$, Dr.Raju Ram Bishnoi ${ }^{3}$, RuchikaGoyal ${ }^{4}$, \\ Dr.AnweshaBiswas ${ }^{5}$ \\ ${ }^{1}$ Postgraduate Department of Conservative Dentistry and Endodontics Maharaja Ganga Singh Dental College \\ Sriganganagar, Rajasthan \\ ${ }^{2}$ Postgraduate Department of Conservative Dentistry and Endodontics, Guru Nanak Dev Dental College, Sunam, Punjab \\ ${ }^{3}$ Postgraduate Postgraduate Department of Oral Medicine and Radiology, Darshan Dental College Udaipur \\ ${ }^{4} 3^{\text {rd }}$ Undergraduate, Guru Nanak Dev Dental College, Sunam, Punjab \\ ${ }^{5}$ Postgraduate Department of Oral Medicine and Radiology
}

Corresponding author: Dr.Yesh Sharma; syesh50@gmail.com

Received 29 December 2019;

Accepted 18 December 2019;

Published 31December 2019

\begin{abstract}
Endodontics is the branch of dentistry in which radiology plays a critical indispensable role. Radiology illuminates what otherwise would be dark and hidden zones and allows the dentists to visualize areas not accessible by other diagnostic means. It is the use of oral radiographs which enables visualization of the bone around the apices of the teeth, as well as the results of the root canal treatments, and as such it has allowed turning endodontics into a scientific professional entity.
\end{abstract}

Keywords: Spiral computed tomography, Tuned aperture computed tomography Fine aperture computed tomography, Magnetic resonance imaging

\section{Introduction}

It is essential to do successful diagnosis of odontogenic and non odontogenicpathoses, treatment of the pulp chamber and canals of the root of a compromised tooth via intracoronal access, biomechanical instrumentation, final canal obturation, and assessment of healing. Imaging serves at all stages in endodontics Preoperative, Intra operative and Post-operative assessment. ${ }^{[1]}$

Preoperatively, Imaging achieves visualization of dental and alveolar hard tissue morphology and pathologic alterations to assist correct diagnosis. It provides information on the morphology of the tooth including location and number of canals, pulp chamber size and degree of calcification, root structure, direction and curvature, fractures, iatrogenic defects, and the extent of dental caries. The effects of per radicular and periapical disease can be determined, including the degree of root resorption and characteristics of periapicalosteolysis. Larger lesions, only determined by imaging, may necessitate adjunctive surgical procedures in addition to conventional intracanal therapy. Diagnostic radiographs help predict the potential for complications, permit root fracture detection, and demonstrate periapical lesions. ${ }^{[2]}$

A "postoperative" radiograph immediately after root canal obturation is made to assess the sealing condensation and containment of the root canal filling material within the root canal system. In cases where periradicular healing is incomplete, it acts as a baseline for assessment of healing in the medium and potentially long term. Imaging is important in evaluating the results of previous therapy, delayed healing, evaluating potential obstacles to retreatment, as well as surgical considerations. ${ }^{[3]}$

\section{Radiographs}

Dr. Wilhelm Conrad Roentgen from Würzberg, Germany, studied rays emitted from a tube in a darkened room; he noticed that some crystals of barium platinocyanide from a table nearby became fluorescent on November 8, 1895.

\section{Roentgen defined the following features of $\mathrm{X}$-rays:}

1. They are able to distinguish between various thicknesses of materials;

2. They cause certain elements to fluoresce;

3. They are made of pure energy with no mass;

4. They go in straight lines; and

5. They are not detectable by human senses.

In modern terms, X-ray radiation is a form of electromagnetic radiation with a wavelength from 0.01 to $10 \mathrm{~nm}$. It is emitted from a metal anode (usually tungsten, molybdenum, or copper) when subjected to a stream of accelerated electrons coming from the cathode. ${ }^{[4]}$

\section{Milestones in the history of dental radiography}




$\begin{array}{lll}* & 1895 & \text { Discovery of X-rays } \\ * & 1896 & \text { First dental radiograph } \\ * & 1901 & \text { First paper on risks of X-radiation } \\ * & 1913 & \text { First prewrapped dental films } \\ * & 1913 & \text { First X-ray tube } \\ * & 1923 & \text { First dental X-ray machine } \\ * & 1947 & \text { Introduction of long-cone Paralleling technique } \\ * & 1957 & \text { First variable kilovoltage dental X-ray machine }\end{array}$

\section{Emergence of digital imaging}

Oral radiographic sensors capable of providing instant images, were introduced in 1984 by Dr. Francis Mouyen from Toulouse, France, and formed the basis for the DRS (Mouyen, 1991). Various digital imaging modalities are available today based on sensors are

- $\quad$ Sensors using charge-coupled device (CCD)

- Complementary metal oxide semiconductor (CMOS)

- Photostimulable phosphor (PSP) technology

Requiring a lower radiation dose and providing instantaneous highresolution digital images make digital radiography especially useful when providing endodontic treatment. Manipulation or processing of the captured image to enhance diagnostic performance makes digital radiography even more versatile in this particular use as it greatly reduces the need to re-expose patients for retakes. ${ }^{[5]}$

Various advanced imaging techniques in use:

Computed tomography

Cone beam computed tomography

Micro computed tomography

Spiral computed tomography

Tuned aperture computed tomography

Fine aperture computed tomography

Magnetic resonance imaging

Ultra sound

Computed Tomography

Computed Tomography (CT) imaging is also known as "CAT scanning" (Computed Axial Tomography). Tomography is from the Greek word "tomos" meaning "slice" or "section" and graphia meaning "describing". It is an imaging technique which produces three-dimensional images of an object by taking a series of twodimensional sectional X-ray images.

- CT was invented in 1972 by British engineer Godfrey Hounsfield of EMI Laboratories, England, and independently by South African born physicist Allan Cormack of Tufts University, Massachusetts.

- The first clinical CT scanners were installed between 1974 and 1976. The original systems were dedicated to head imaging only, but "whole body" systems with larger patient openings became available in 1976.

\section{Computed tomography scanners}

- Essentially, CT scanners consist of a gantry which contains the rotating X-ray tube head and reciprocal detectors.

- In the centre of the gantry, there is a circular aperture, through which the patient is advanced.
W.C. Roentgen

O. Walkhoff

W.H. Rollins

Eastman Kodak Company

W.D. Coolidge

Victor X-ray Corporation

F.G. Fitzgerald

General Electric

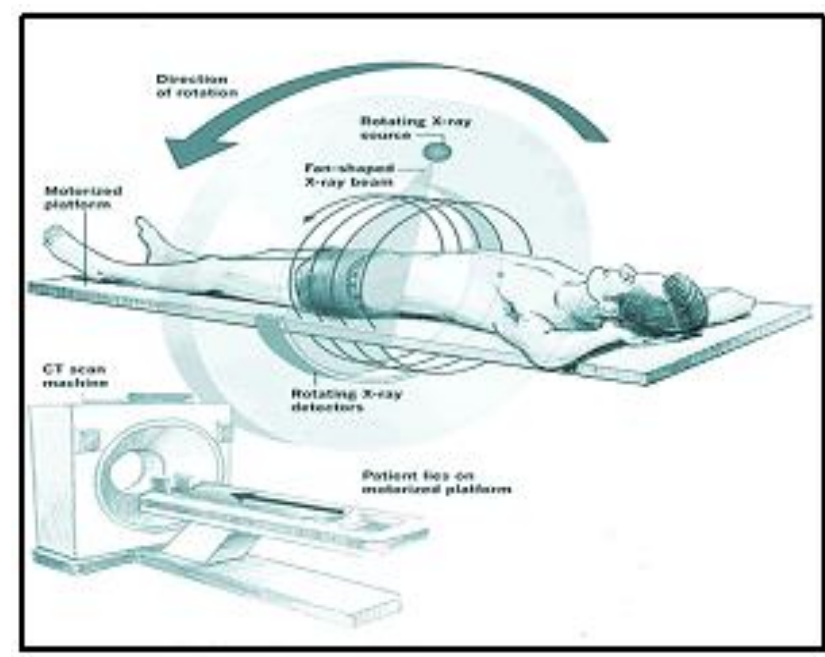

Fig. 1

- The tube head and reciprocal detectors within the gantry either rotate synchronously around the patient, or the detectors take the form of a continuous ring around the patient and only the X-ray source moves within the gantry.

- The data from the detectors produce an attenuation profile of the particular slice of the body being examined. The patient is then moved slightly further into the gantry for the next slice data to be acquired. The process is repeated until the area of interest has been scanned fully. [6]

Reconstructed three-dimensional CT image, sagittal, axial and coronal reconstructed slices from a CT scan.

\section{Applications of CT in Endodontics}

1. Location of inferior alveolar canal

2. Surgical retreatment

3. Diagnosis of odontogenic pain

4. Non-surgical retreatment

5. Proximity of anatomic structures

\section{Cone beam computed tomography}

Cone beam computed tomography or digital volume tomography is an extra-oral imaging system which was developed in thelate1990s to produce three-dimensional scans of the maxillo-facial skeleton at a considerably lower radiation dose than CT. 


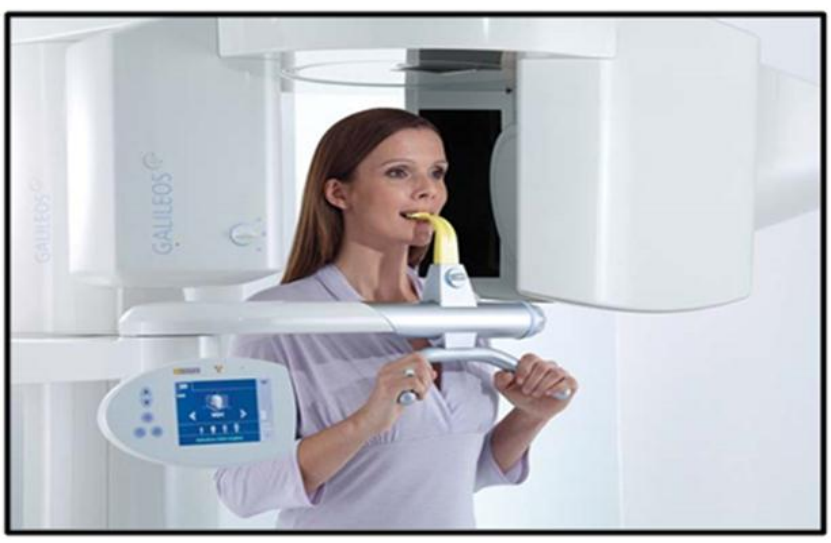

Fig.2

\section{CBCT scanners}

CBCT is accomplished by using a rotating gantry to which an $\mathrm{X}$ ray source and detector are fixed. A divergent pyramidal- or coneshaped source of ionizing radiation is directed through the middle of the area of interest onto an area X-ray detector on the opposite side of the patient. The X-ray source and detector rotate around a fixed fulcrum within the region of interest (ROI).

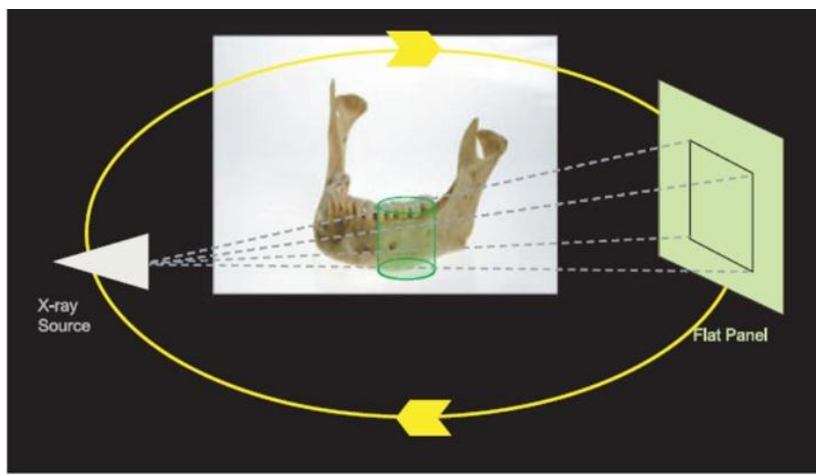

Fig. 3

During the exposure sequence hundreds of planar projection images are acquired of the field of view (FOV) in an arc of at least $180^{\circ}$. In this single rotation, CBCT provides precise, essentially immediate and accurate 3D radiographic images.

As CBCT exposure incorporates the entire FOV, only one rotational sequence of the gantry is necessary to acquire enough data for image reconstruction. CBCT is a complementary modality for specific applications rather than a replacement for 2D imaging modalities. ${ }^{[7]}$

Table 1: Comparison of the effective dosages and background equivalent of different sources of dental radiation

\begin{tabular}{|l|l|l|}
\hline Radiographic Source & Effective Dose (Msv) & Dose As \% Annual Background Radiation \\
\hline CBCT & 7.3 & 0.2 \\
3d accuitomo (11/2 inch)[40] i-cat b (12 inch fov) [45] i- & 134.8 & 5.4 \\
cat b (9 inch fov)[45] & 68.7 & 1.9 \\
\hline conventional CT[39] & 1400 (maxilla) & 38.9 \\
& 1320 (mandible & 36.7 \\
\hline Conventional radiography Periapical[45] Panoramic[46] & 5 & 0.14 \\
& 6.3 & 0.2 \\
\hline
\end{tabular}

The technology has been widely accepted and is now being used for research and clinical purposes-

$\begin{array}{ll}\text { - } & \text { Detection of apical periodontitis } \\ \text { - } & \text { Early detection of periapical pathology: } \\ \text { - } & \text { Accurate determination of prognosis of treatment: } \\ \text { - } & \text { Retreatment } \\ \text { - } & \text { Assessment of dental trauma } \\ \text { - } & \text { Detection of horizontal root fractures } \\ \text { - } & \text { Treatment of large destructive lesions } \\ \text { - } & \text { Detection of root resoption }\end{array}$

Tuned aperture computed tomography (TACT)

Tuned aperture computed tomography works on the basis of tom synthesis (Webber\& Messura1999). A series of 8-10radiographic images are exposed at different projection geometries using a programmable imaging unit, with specialized software to reconstruct a three-dimensional dataset which may be viewed slice by slice (Fig. 4). ${ }^{[8]}$

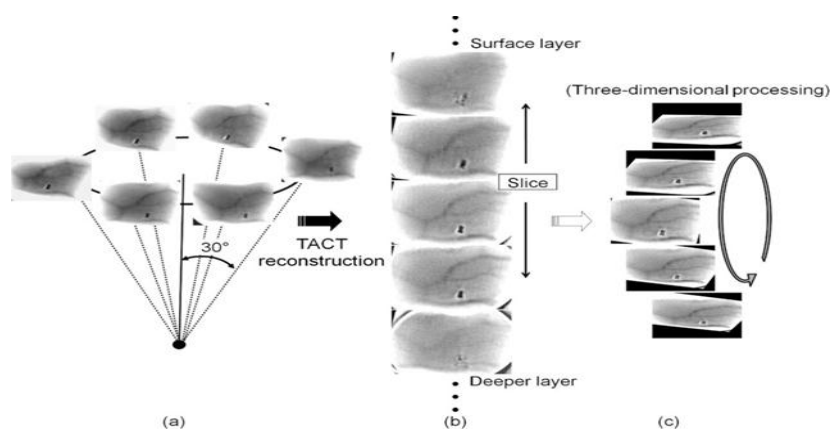

Fig.4
Spiral computed tomography

Spiral computed tomography is a computed tomography technology involving movement in a helical pattern for the purpose of increasing resolution. Most modern hospitals currently uses spiral CT scanners.

In cone-beam computed tomography (commonly abbreviated CBCT), the $\mathrm{x}$-ray beam is conical. Helical (or spiral) cone beam computed tomography is a type of three-dimensional computed tomography(CT) in which the source (usually of x-rays) describes a helical trajectory relative to the object while a two-dimensional array of detectors measures the transmitted radiation on part of a cone of rays emanating from the source. ${ }^{[9]}$

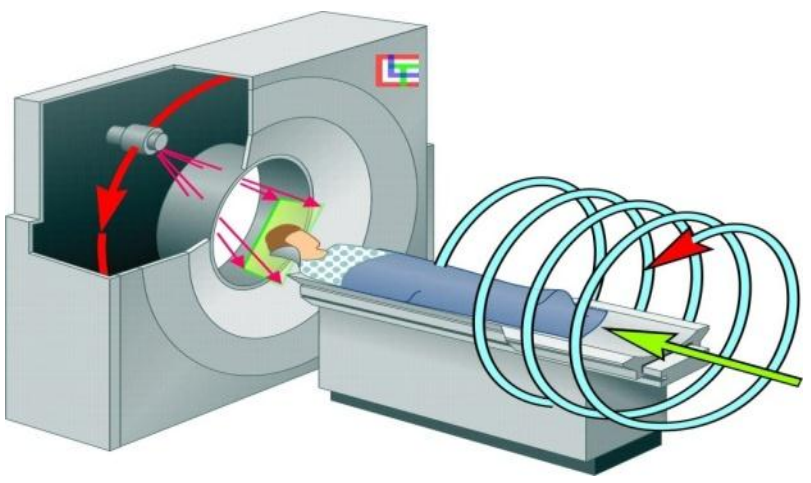

Fig.5

- In practical helical cone beam x-ray CT machines, the source and array of detectors are mounted on a rotating gantry while the patient is moved axially at a uniform rate. 
- $\quad$ Earlier x-ray CT scanners imaged one slice at a time by rotating source and one dimensional array of detectors while the patient remained static. The helical scan method reduces the x-ray dose to the patient required for a given resolution while scanning more quickly. This is however at the cost of greater mathematical complexity in the reconstruction of the image from the measurements. ${ }^{[10]}$

\section{Micro Computed Tomography}

High-resolution micro-computed tomography is an innovative technology with several applications in endodontic research and education.

Using CT, the investigators were able to demonstrate anatomical configuration of teeth, but the spatial resolution of 0.6 mmwas found to be insufficient to allow for detailed analysis of root anatomy and structures. The authors concluded that conventional CT offered only limited application in endodontics due to its high radiation dose, time consumption, cost, insufficient resolution, and inadequate computer software capability.

Technological advancements allowed for the introduction of a miniaturized form of traditional CT, the micro-CT for use in nonclinical settings by kak and Stanley in 1988.Micro-CT applies comparable principles to those of conventional CT, but the threedimensional reconstructions of small objects, such as teeth, are developed to a resolution of within a few microns $(<2 \mu \mathrm{m}) .^{[11]}$

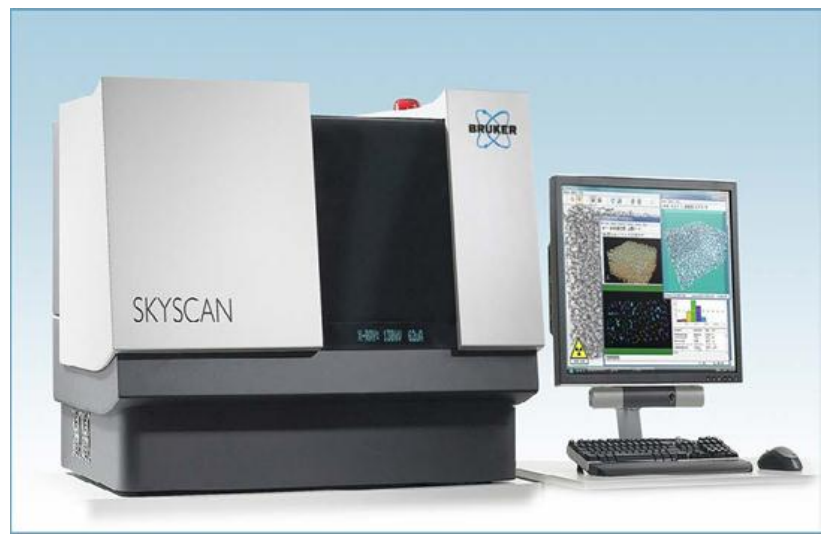

Fig.6

\section{Applications in Endodontics}

As a non-destructive imaging tool, micro-CT may be applied to assess an object many times, allowing it to remain unaltered for further experimentation and future scans. The three-dimensional images gather considerable data, allowing for both qualitative and quantitative evaluation of the sample. These characteristics make micro-CT a desirable tool for in vitro studies that evaluate root canal morphology and procedures of root canal preparation and obturation. Thus, a scanned tooth can be analyzed along its length to acquire data for calculating areas and volumes before and after endodontic procedures. ${ }^{[12]}$

The data offered by micro-CT technology can lead to clinical applications such as development of new techniques, comparative analysis of existing approaches in endodontic treatment, and enhancement of dental education in preclinical and clinical stages.

\section{Ultrasound}

The first application of acoustic echoes for object localization took place under water in search for the wreck of the Titanic in 1912.Seventy years ago Dussik introduced the use of ultrasounds as a diagnostic tool in the medical field to evaluate a cerebral pathosis. Since that far, 1942 diagnostic ultrasounds have gained more and more importance, and now a-days, there is no diagnostic field in medicine in which ultrasonic imaging does not find a specific indication. ${ }^{[13]}$

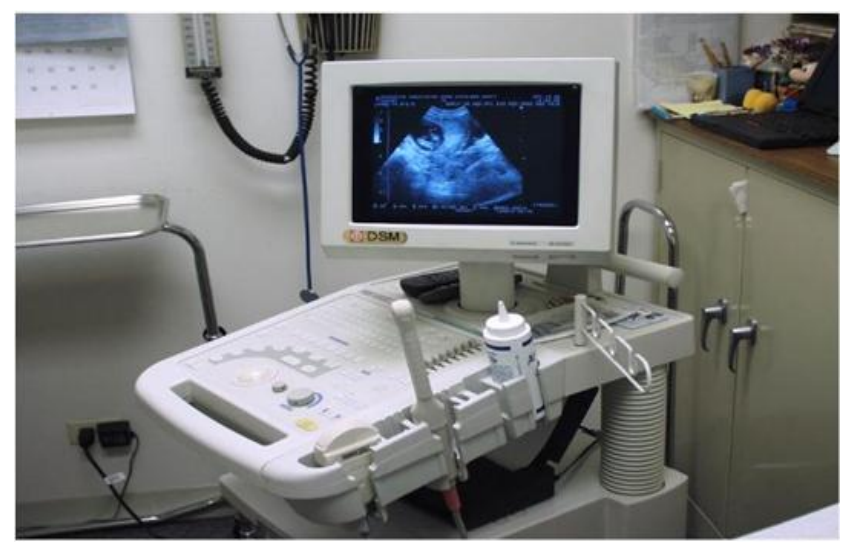

Fig.7

\section{Echo Color Power Doppler}

To better evaluate bone lesions in the jaws, and especially to reduce the artifacts in fluid structures (cysts), these are simultaneously insonated with converging boundless US waves sent from different directions (COMPOUND TECHNIQUE). ${ }^{[14]}$

The color-power Doppler (CPD) flowmetry applied to the US examination allows the accurate evaluation of the blood flow within a given tissue, and it is based on the "Doppler effect" discovered in 1842 (Fleischer and Emerson, 1993) as a consequence of the frequency shift that occurs in the reflected sound waves when the US waves are directed toward the moving blood cells. ${ }^{[15]}$

The color Doppler is the technique used for visualizing the presence and velocity of the blood flow within an image plane. It measures the Doppler shifts in the volumes located in an image plane and shows the flow in the form of color spots superimposed on the gray-scale image. Blood vessels moving toward the transducer appear as red (positive Doppler shifts), while vessels moving away from the transducer appear as blue (negative Doppler shifts).

The higher velocities of the flower represented in bright shades of the same colors (red and blue); the scheme of the color which encodes the frequency shifts is indicated by the color bar to the side of the screen /image

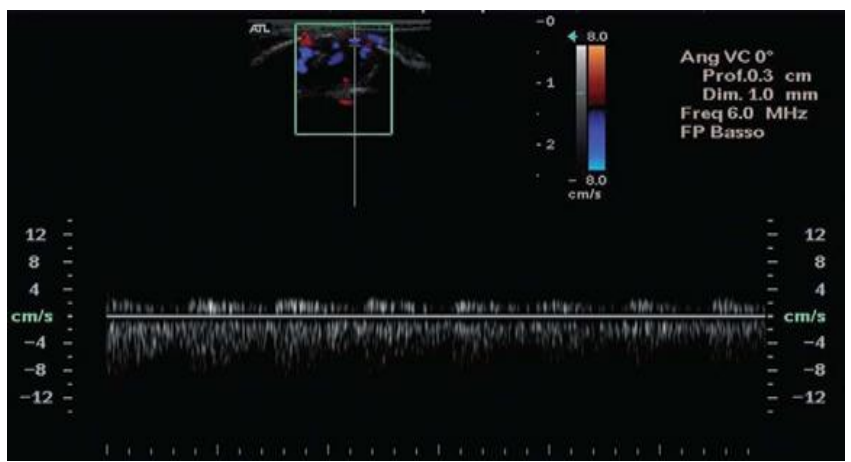

Fig.8

Figure 19.1 Echo-color Doppler applied to the examination of a periapical lesion showing the Doppler signal in the lower portion of the figure, and the color Doper in the format of colored spots superimposed on the lesion, in the upper part of the figure (framed). 


\section{Applications in Endodontics}

In the field of endodontics, the echo-CDP discloses the vascular map around and within a lesion and shows the direction of blood flow.

US real-time imaging has been applied to the endodontic field for the diagnosis, evaluation, and follow-up of bone lesions in the jaws.
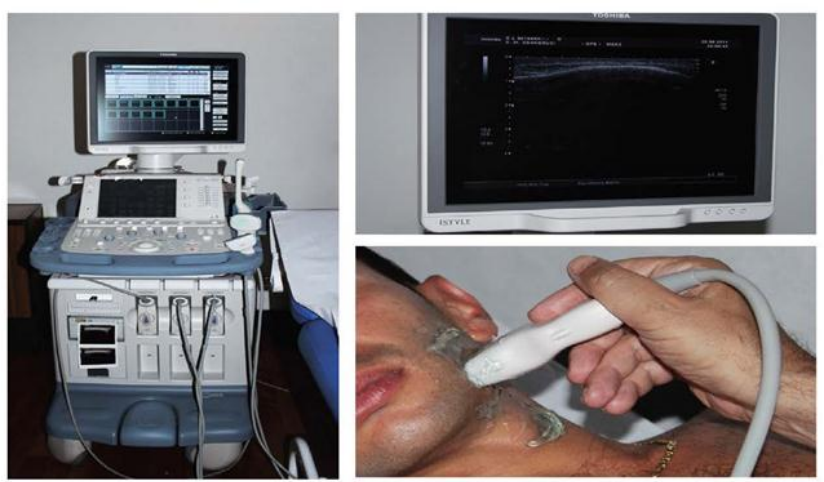

Fig.9

\section{Future scope of ultrasound in endodontics:}

As an opening to the near future, US have promise for study and early diagnosis of a variety of dental diseases:

1) Caries, cracks, and fractures by measuring enamel thickness and track changes in enamel thickness over space and time,

2) Periodontal disease, by qualitatively assessing the periodontium,

3) Bone characterization for implant treatment plan and osteoporosis, via noninvasive measurements during routine visits.

\section{Magnetic Resonance imaging}

Magnetic resonance imaging is a specialized imaging technique which does not use ionizing radiation. It involves the behaviour of hydrogen atoms (consisting of one proton and one electron) within a magnetic field which is used to create the MR image.

Paul Lauterbur described the first magnetic resonance image in 1973 and PETER MANSFIELD further developed use of magnetic field and mathematical analysis of the signals for image reconstruction. MRI has been available since 1984.

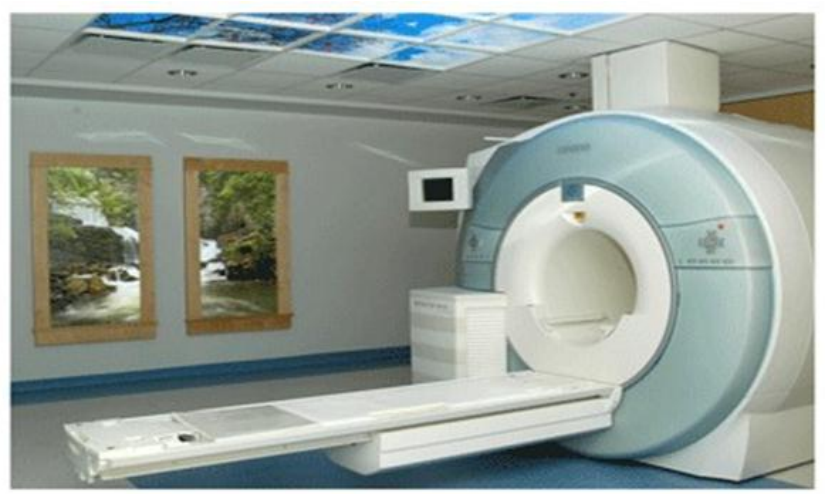

Fig.10

\section{Applications in Endodontics:}

- The application of MRI to dentistry has more often involved the temporomandibular joints and salivary glands.
- Its use has also been reported on the assessment of the jaw bones prior to implant surgery, and on the differential diagnosis of lesions in the mandibular and maxillary bones.

Swift (sweep imaging with Fourier transformation) imaging MRI is also feasible for looking at dental carious lesions in three dimensions and no artefacts are created by metallic restorations within this exam, but it is difficult to distinguish the hard tissues of the tooth within the MRI scans because of their scarce water content. To overcome these problems, a new MRI technique has been developed very recently: the SWIFT (Sweep Imaging with Fourier Transformation) imaging. ${ }^{[15]}$

\section{Conclusion}

Even with the best intentions and refined technique, images acquired using conventional intra-oral radiographs reveal information in two-dimensions only (height and width) limiting the valuable information in the third dimension i.e., "depth of the lesion". Besides this, the inherent problems of positioning image receptors in the ideal position in relation to the anatomical area of interest, anatomic noise, under or over-estimation of actual healing or failure of endodontic treatment limits the assessment of true nature of the endodontic lesions. In such situations, advanced diagnostic aids like CBCT, CT, micro CT are very helpful in visualizing the lesion three dimensionally. Especially CBCT technology is improving at a rapid pace because of the improved diagnostic data provided and therefore appropriate decision making for the management of complex endodontic problems. But it is also essential to remember that it uses ionizing radiation and therefore it is not without risk. So, endodontic cases should be judged individually and CBCT should only be considered in situation where information from conventional imaging systems does not yield an adequate amount of information to allow appropriate management of the endodontic problem.

\section{References}

[1] Grossman, L.I. (1982) A brief history of endodontics. J Endod, 8, Special Issue, 36.

[2] R. E. Walton, "Diagnostic imaging A. endodontic radiography," in Ingles' Endodontics, J. I. Ingle, L. K. Bakland, and J. C. Baumgartner, Eds., p. 554, BC Decker, Hamilton, Canada, 6th edition, 2008.

[3] V. E. Rushton, K. Horner, and H. V. Worthington, "Screening panoramic radiology of adults in general dental practice: radiological findings," British Dental Journal, vol. 190, no. 9, pp. 495- 501, 2001.

[4] Webber RL, Messura JK (1999) An in vivo comparison of digital information obtained from tuned-aperture computed tomography and conventional dental radiographic imaging modalities. Oral Surgery, Oral Medicine, Oral Pathology, Oral Radiology and Endodontology 88, 239-47.

[5] Arai Y, Honda K, Iwai K, Shinoda K (2001) Practical model '3DX' of limited cone-beam Xray CT for dental use. International Congress Series 2001, 713-8.

[6] Mozzo P, Procacci C, Tacconi A, Martini PT, Andreis IA (1998) A new volumetric CT machine for dental imaging based on the cone-beam technique: preliminary results. European Radiology 8, 1558-64. 
[7] De Santis, R., Mollica, F., Prisco, D., Rengo, S., Ambrosio, L., and Nicolais, L. (2005) A 3D analysis of mechanically stressed dentin-adhesive-composite interfaces using X-ray micro-CT. Biomaterials, 26, 257270

[8] Nielsen, R.B., Alyassin, A.M., Peters, D.D., Carnes, D.L., and Lancaster, J. (1995) Microcomputed tomography: an advanced system for detailed endodontic research.JEndod, 21, 561-568.

[9] Dowker, S.E., Davis, G.R., and Elliott, J.C. (1997a) Xray microtomography: nondestructive three-dimensional imaging for in vitro endodontic studies. Oral Surg Oral Med Oral Pathol Oral RadiolEndod, 83, 510-516.

[10] Jung, M., Lommel, D., and Klimek, J. (2005) The imaging of root canal obturation using micro-CT. IntEndod J, 38, 617-626
[11] Cheung, L.H. and Cheung, G.S. (2008) Evaluation of arotary instrumentation method for Cshaped canals with micro-computed tomography. J Endod, 34, 1233-1238.

[12] Min, Y., Fan, B., Cheung, G.S., Gutmann, J.L., and Fan, M. (2006) C-shaped canal system in mandibular second molars. Part III: The morphology of the pulp chamber floor. J Endod, 32, 1155-1159.

[13] Peters, O.A., Laib, A., Rüegsegger, P., and Barbakow, F. (2000) Three-dimensional analysis of root canal geometry by high-resolution computed tomography. J Dent Res, 79, 1405-140.

[14] Paque, F., Barbakow, F., and Peters, O.A. (2005) Root canal preparation with Endo-Eze AET: changes in root canal shape assessed by micro-computed tomography. IntEndod J, 38, 456-464.

[15] Hammad, M., Qualtrough, A., and Silikas, N. (2009) Evaluation of root canal obturation a threedimensional in vitro study. J Endod, 35, 541-544. 